\title{
Synthesis and Structural Characterization of Glycidyl Ethyl Polyhedral Oligomeric-Sil-Sesquioxane (GE-POSS) Coated SiC Nanoparticles
}

\author{
V.K.Rangari,*, R.E.Rabby and S. Jeelani, \\ ${ }^{1}$ Materials Science and Engineering, Center for advanced Materials, Tuskegee University, \\ Tuskegee, AL, 36088
}

It is well known in the literature that $\mathrm{SiC}$ particles have a tendency to oxidize when exposed to the air $[1,2]$. These contaminations or oxidization is mostly by silica, oxicarbide or free carbon. Interestingly, formation of these coherent layers of $\mathrm{SiO}_{2}$ onto $\mathrm{SiC}$ was considered one of the reasons for high temperature resistance property of $\mathrm{SiC}$, as $\mathrm{SiO}_{2}$ restrain from supplementary oxidation on its surface [1]. These articulate layers is likely to be formed by breaking bonding between $\mathrm{Si}$ and $\mathrm{C}$ in the presence of oxygen to form bonding among $\mathrm{Si}, \mathrm{C}$, and oxygen. Polyhedral Oligomeric Silsesquioxane (POSS), which is the organic-inorganic hybrid nanomaterial of 0.5-3.0 $\mathrm{nm}$ in dimension, has a wide range of application in nanocomposites such as coating materials or surface modifiers, catalysts, membrane materials etc. by grafting or blending or homopolymerization or copolymerization into thermoplastic and thermoset polymers with a remarkable enhancement in mechanical and thermal properties [13-20]. Surface characterization, and modification of a nanomaterial is very essential to understand and use them in the harsh environment. In this research work, partial Oxidation on SiC surface was performed by coating $\mathrm{SiC}$ with GlycidylEthyl (GE) POSS using sonochemical synthesis technique. Sonochemical oxidation of as received $\mathrm{SiC}$ nanoparticles were also studies for comparison. The as-received nanoparticles and their hybrid nanoparticles were characterized using high-resolution transmission electron microscopy (HR-TEM), X-ray photoelectron spectroscopy (XPS) and Fourier transform infrared spectrum (FTIR). These results reveal that covalent bonding between $\mathrm{SiC}$ and GE-POSS was formed. This bonding was supported from the surface structural and chemical composition, binding energy.

Corresponding author:

*Dr.Vijaya Kumar Rangari

Email: rangariv@mytu.tuskegee.edu

Fax: 3347272286 

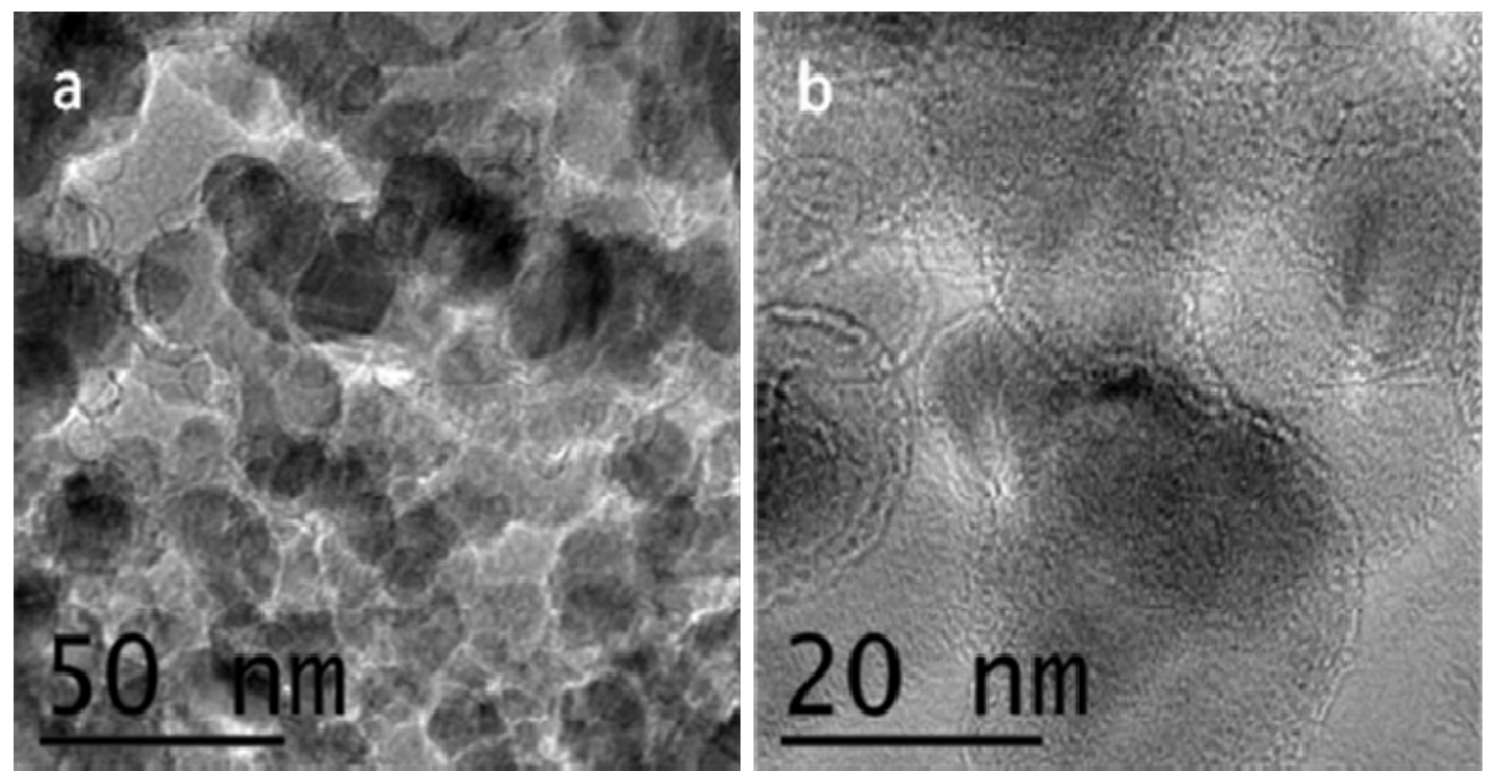

Figure 1. TEM micrograph of POSS coated $\mathrm{SiC}$ hybrid nanoparticles

The morphology and sizes of GE-POSS coated SiC hybrid nanoparticles were examined by HRTEM and shown in Figure 1. This figure illustrate the TEM images of POSS GE coated SiC where dark spherical particles were surrounded by shaded layer of GE POSS. The contrast within the particles from figure 1 is due to the coating of GE POSS onto SiC nanoparticles where the dark particle, which are $\mathrm{SiC}$ (15 to $30 \mathrm{~nm}$ in diameter) with the coating of thin layer of GEPOSS. These results were also confirmed with XPS and FTIR studies.

\section{References:}

[1] J.A.Costello et al., J Am Ceram Soc, 69, (1986)674-681.

[2] C. Onneby et al., J. Vac. Sci. Technol. A, 15(3), (1997) 1597-1602.

[3] M. Iijima et al., J. Phys. Chem. C, 112, (2008) 11786-11790.

[4] G. Xi et al., J. Phys. Chem. B, 109, (2005) 13200-13204.

[5] Z. Guo et al., Composites Science and Technology, 68, (2008) 164-170. 